\title{
Psicoterapia de Crianças: Desenvolvimento da Versão em Português do Child Psychotherapy Q-Set
}

\author{
Vera Regina Rohnelt Ramires ${ }^{1}$ \\ Universidade do Vale do Rio dos Sinos \\ Celeste Schneider \\ San Francisco Center for Psychoanalysis, CA, USA
}

\begin{abstract}
RESUMO - A literatura científica reconhece a necessidade de aprofundar o conhecimento sobre o processo terapêutico de crianças, compreendendo os mecanismos pelos quais se produzem mudanças. Para isso, precisamos de instrumentos que viabilizem tais investigações. O objetivo deste estudo foi traduzir e adaptar para o português brasileiro o Child Pychotherapy $\mathrm{Q}-\mathrm{Set}(\mathrm{CPQ})$, instrumento que visa à análise do processo psicoterapêutico de crianças. O estudo foi desenvolvido em quatro etapas, avaliando-se a equivalência entre a versão traduzida e o instrumento original em quatro áreas: equivalência semântica, idiomática, experiencial e conceitual. Constatou-se que a versão em português do CPQ apresentou boa equivalência com a original e a fidedignidade interavaliadores foi satisfatória. As possibilidades de utilização oferecidas pelo CPQ foram discutidas.
\end{abstract}

Palavras-chave: psicoterapia da criança, pesquisa em psicoterapia, processos terapêuticos, tradução, adaptação de instrumento

\section{Children's Psychotherapy: Development of a Portuguese version of the Child Psychotherapy Q-Set}

\begin{abstract}
Scientific literature recognizes the need to increase knowledge on child psychotherapeutic process, understanding the mechanisms by which changes occur. In order to do this, we need instruments that will make possible such investigations. The aim of this study was to translate and adapt to Brazilian Portuguese the Child Psychotherapy Q-Set (CPQ), an instrument that aims to analyze the psychotherapeutic process of children. The study was conducted in four stages, assessing the equivalence between the translated version and the original instrument in four areas: semantic equivalence, idiomatic, experiential and conceptual. It was found that the Portuguese version of the CPQ presented good equivalence with the original and the interrater reliability was satisfactory. The possibilities provided by the CPQ were discussed.
\end{abstract}

Keywords: child psychotherapy, psychotherapy research, therapeutic processes, translating, instrument adaptation

A psicoterapia de crianças é um tratamento especializado para a abordagem das dificuldades emocionais, sociais e/ ou comportamentais dessa população. Parte da premissa central de que é possível e pode ser benéfica a comunicação com conteúdos internos, emocionais e imaginários da subjetividade, que são muito ativos durante a infância e permanecem influentes ao longo da vida, mesmo quando não se está consciente deles (Midgley, 2007).

É unânime na literatura científica a existência de uma lacuna entre pesquisa em psicoterapia e prática clínica. Inúmeras modalidades de tratamento não têm sido pesquisadas quanto à sua eficácia ou efetividade, tampouco quanto aos seus mecanismos de mudança. Conforme Kazdin (2008), dezenas de modalidades de tratamento são oferecidas para a população infanto-juvenil, e esse número continua crescendo. A vasta maioria nunca foi estudada, seja em ensaios controlados ou não. Nem todos esses tratamentos derivam de uma teoria explícita sobre etiologia ou mudança terapêutica. Fonagy (2009) questiona se todos esses modelos seriam necessários e, ainda, se todos eles estão trabalhando

1 Endereço para correspondência: Rua Luzitana 1148, Apto 1002, Porto Alegre, RS, Brasil. CEP: 90.520-080 E-mail: verareginaramires@ gmail.com com base em diferentes princípios. Entende que não estamos testando essas alternativas de modo significativo e de maneira a permitir uma discriminação e seleção entre tantas opções.

Kazdin (2000) entende que a maior limitação da pesquisa em psicoterapia é a escassez de estudos dedicados a explicar por que e como os tratamentos funcionam. Na sua revisão de mais de 200 estudos sobre psicoterapia de crianças - que incluiu a abordagem psicodinâmica, a cognitivocomportamental e outras - identificou que menos de 3\% desses estudos examinaram o processo da psicoterapia em relação aos resultados. Assinalou, entretanto, que a menos que a compreensão do processo psicoterapêutico seja incorporada à investigação dos resultados, o progresso será limitado.

Compreender por que e como as mudanças acontecem como resultado de uma intervenção terapêutica tem o potencial de auxiliar a identificar os ingredientes ativos ou mecanismos de mudança que estão na base de uma psicoterapia bem sucedida (Fonagy, 2003; Kazdin, 2009; Midgley, 2007). A compreensão dos processos que explicam a mudança terapêutica permite otimizar a mudança, identificar diferentes ou melhores estratégias e identificar os mediadores e os moderadores do tratamento. Dois pacientes 
com as mesmas dificuldades, recebendo o mesmo tipo de psicoterapia, com o mesmo terapeuta, podem responder positivamente por diferentes razões. $\mathrm{O}$ conhecimento da complexidade dos mecanismos de mudança é importante para assegurar que o melhor cuidado e uma "variação ótima do tratamento" estão sendo oferecidos (Kazdin, 2009).

A análise do processo terapêutico com todas as suas variáveis e que capture todos os seus fenômenos e nuances constitui, ainda, um desafio para os pesquisadores e clínicos. A pesquisa nesse campo levanta a questão das medidas, instrumentos ou procedimentos que viabilizem seu desenvolvimento.

Com o objetivo de responder a essa demanda, Enrico Jones (2000) desenvolveu o Psychotherapy Process Q-set $(P Q S)$, procedimento que visa compreender o papel dos diferentes fatores envolvidos no processo terapêutico. Um dos seus pressupostos era que, para usar dados clínicos e testar constructos teóricos, seria necessário, primeiro, estabelecer que certos tipos de fenômenos observáveis ocorrem e então avaliá-los. Os fenômenos clínicos devem ser observáveis intersubjetivamente, "o que significa dizer que diferentes juízes podem independentemente concordar sobre sua ocorrência e suas características" (Jones, 2000, p. 256).

É conhecida no campo das psicoterapias a existência de discordâncias sobre a interpretação ou o significado do mesmo material clínico. Por isso, é crucial que uma metodologia de pesquisa estabeleça a extensão do consenso entre juízes sobre a presença e a natureza dos fenômenos clínicos, ou seja, sua confiabilidade (Jones, 2000). O desenvolvimento do PQS buscou responder a esse desafio e oferecer uma linguagem básica para descrição e classificação do processo terapêutico de uma forma adequada para análise quantitativa também.

O PQS tem sido reconhecido pela sua efetividade em capturar a complexidade do processo terapêutico com adultos e tem contribuído para informar sobre esse processo e sua relação com o resultado do tratamento. Conforme Schneider, Pruetzel-Thoma e Midgley (2009), "o trabalho de Jones e colegas tem desafiado noções aceitas sobre várias modalidades de tratamento, revitalizado o uso do delineamento de estudo de caso único na pesquisa em psicoterapia e promovido o estudo de psicanálises e psicoterapias de longa duração" (p. 74). O PQS foi traduzido e adaptado para o português por Serralta, Nunes e Eizirik (2007).

Para o estudo do processo terapêutico de crianças, foi desenvolvido por Celeste Schneider e Enrico Jones, com base no PQS, o Child Psychotherapy Q-Set (CPQ; Schneider, 2003; Schneider \& Jones, 2006, 2012). O CPQ permite a análise do processo terapêutico de crianças de 3 a 13 anos de idade e é composto por 100 itens que representam uma ampla gama das características da psicoterapia de crianças. Um subconjunto de itens descreve as atitudes, sentimentos, comportamento ou experiência da criança (e. g., CPQ Item 29 - A qualidade do jogo da criança é fluída, absorta versus fragmentada ou esporádica). Um segundo subconjunto reflete as ações e atitudes do terapeuta (e. g., CPQ Item 2 O terapeuta comenta sobre o comportamento não verbal da criança - postura corporal, gestos). O terceiro subconjunto de itens busca capturar a natureza da interação da díade ou a atmosfera do encontro (e. g., CPQ Item 27 - Há um foco em ajudar a criança a planejar seu comportamento fora da sessão).

Os itens do CPQ resultaram de uma seleção baseada em uma extensa revisão de literatura sobre psicoterapia de crianças, que inclui métodos de tratamento validados empiricamente e abordagens psicanalíticas (Schneider \& Jones, 2006; Schneider, et al., 2009). Os itens foram baseados também em instrumentos sobre processo de psicoterapia existentes e adaptações da forma adulta PQS.

Os avaliadores, de maneira independente, assistem ao vídeo de cada sessão e classificam os itens do CPQ de acordo com o quanto foram característicos ou não daquela sessão (Schneider \& Jones, 2006, 2012; Schneider et al., 2009). Trata-se de um processo meta-analítico. Portanto, é mais do que a mera identificação do comportamento, implicando um movimento por parte do avaliador entre a observação e a formulação. Na medida em que o avaliador classifica os itens do CPQ, um mosaico dos padrões terapêuticos começa a emergir, mosaico este que auxiliará a descrever o processo interativo do terapeuta e da criança.

O CPQ tem sido utilizado em estudos desenvolvidos nos Estados Unidos e Inglaterra, visando explorar em profundidade a natureza do processo terapêutico e sua relação com o resultado (Schneider, Midgley, \& Duncan, 2010; Schneider et al., 2009), a elaboração de protótipos da psicoterapia de crianças (Goodman, Midgley, \& Schneider, 2016) e a identificação e análise das estruturas de interação no processo terapêutico (Goodman \& Athey-Lloyd, 2011). As análises qualitativas e quantitativas possibilitadas pelo procedimento assim como a investigação do processo ao longo do tempo no interior de cada psicoterapia e entre diferentes tratamentos são recursos que oferecem importantes e inéditos vértices de observação.

Dessa forma, o objetivo deste estudo foi traduzir e adaptar para o português brasileiro o CPQ. Ele é parte integrante de um projeto mais amplo denominado Processo da Psicoterapia Psicanalítica de Crianças: Uma Análise em Relação ao Protótipo e aos Resultados, uma investigação intensiva e sistemática de duas psicoterapias psicanalíticas de crianças em idade escolar, que envolveu a aplicação do CPQ às sessões do primeiro ano dessas psicoterapias e sua análise, relacionando-a às mudanças e resultados obtidos durante esse mesmo período.

\section{Método}

\section{Instrumento}

O CPQ, da mesma forma que o PQS, é baseado na Q-metodologia, desenvolvida por William Stephenson (1982). Trata-se de uma técnica que oferece meios convenientes para organizar dados em termos de sua representatividade. Os dados a serem ordenados podem se referir a um constructo particular, uma pessoa ou situação que está sendo descrita (Schneider, 2003; Schneider et al., 2009). Não existe um conjunto Q Padrão. O objetivo é oferecer um conjunto de elementos que descreva da melhor 
maneira possível as dimensões do fenômeno em estudo e suas variações (Serralta et al., 2007).

O CPQ pode ser utilizado para analisar diferentes abordagens terapêuticas com crianças. Os 100 itens do instrumento são apresentados em cartões individuais. Para permitir maior confiabilidade e minimizar possíveis variações na interpretação dos itens, um manual de codificação oferece definições claras, descrições e exemplos operacionais de cada item, refletindo características que podem ser identificadas usando videotapes das sessões.

Os itens são avaliados uns em relação aos outros e não isoladamente. Os 100 cartões contendo os itens devem ser ordenados em nove categorias, colocando-se em uma extremidade aqueles cartões cujas afirmações são mais características ou salientes no material analisado naquela sessão em particular e na outra extremidade os cartões menos característicos. Um número fixo de itens deve ser colocado em cada categoria, constituindo-se como um método de ordenamento forçado, que resulta em uma distribuição normal. O maior número de itens deve ser colocado no centro (18), refletindo afirmações não descritivas ou menos descritivas do que aquelas nas extremidades (5).

A linguagem e o procedimento de classificação do CPQ permitem extrair e descrever a dinâmica que guia a interação paciente e terapeuta. Cada sessão de psicoterapia deve ser avaliada por, pelo menos, dois juízes, que devem ser psicoterapeutas experientes e treinados para uso do instrumento. Os avaliadores, de maneira independente, realizam a avaliação do videotape da sessão em um procedimento de ordenação (Q-sorting), que tanto identifica temas salientes observáveis como reúne princípios organizadores subjacentes de uma sessão (Jones, 2000; Schneider et al., 2009). A terminologia teórica é reduzida ao mínimo, permitindo retratos da interação paciente e terapeuta teoricamente insaturados. As codificações dos juízes devem atingir um índice de concordância (alpha de Cronbach) de pelo menos 0,70 pelo método de correlações intraclasses. Não sendo atingido esse resultado, um terceiro avaliador codifica a mesma sessão, até atingir-se o índice exigido.

O projeto de desenvolvimento do instrumento se estendeu por quatro anos (1999 a 2003), incluiu três estudos e envolveu um processo recursivo de construção dos itens, estudos pilotos para obter validade clínica e reconstruções (Schneider, 2003). Os itens iniciais foram revisados por psicoterapeutas experientes, que representavam vários pontos de vista teóricos (incluindo psicoterapia psicanalítica e cognitivocomportamental), oferecendo feedback sobre a clareza dos itens, redundância, abrangência e representatividade.

No terceiro estudo, para estabelecer a validade dos itens entre diferentes crianças em vários modelos de psicoterapia, sete clínicos experientes representando diferentes abordagens teóricas (cognitivo-comportamental, psicodinâmica e psicanálise) revisaram o processo em 20 videotapes de terapias de criança que incluíam tratamentos psicanalíticos, psicodinâmicos e cognitivos-comportamentais (tratamentos desenvolvidos na University of California at Berkeley Psychology Clinic, Massachusetts General Psychology Clinic e Anna Freud Centre, London; tempo de experiência dos clínicos variando de 2 a 25 anos). De acordo com Schneider (2003) e Schneider et al. (2009), uma matriz de correlação dos itens dessas avaliações identificou itens que demonstraram pouca variação entre os casos e que eram redundantes, conduzindo a reformulações ou substituições. Consequentemente, cada item do CPQ foi elaborado para cobrir um aspecto único do processo terapêutico, não duplicado por nenhum outro item, visando garantir uma variabilidade máxima entre sessões e entre diferentes pacientes.

Diversas investigações com o PQS (instrumento mais antigo e correlato do CPQ) demonstraram que ele apresenta boa fidedignidade interavaliadores (Jones, 2000; Jones, Cumming, \& Horowitz, 1988; Jones \& Pulos, 1993), validade de construto (Jones et al., 1988) e validade discriminante (Jones \& Pulos, 1993). De acordo com Serralta et al. (2007), a validade fatorial é irrelevante, uma vez que a medida supõe independência entre os itens. O estudo de Jones et al. (1988) evidenciou ausência de estrutura fatorial, o que seria bastante desejável do ponto de vista da metodologia Q, conforme testemunham Serralta et al.

A fidedignidade e validade do CPQ também vêm sendo demonstradas por diferentes estudos. Sua validade discriminante foi demonstrada entre dois conjuntos de sessões de psicoterapia psicodinâmica e terapia cognitivocomportamental (Schneider et al., 2009). Também para examinar a validade discriminante do CPQ, foi conduzido um estudo com 32 terapeutas experientes (mais de 15 anos de experiência) representando diferentes orientações teóricas (psicodinâmica, cognitivo-comportamental e abordagem baseada na função reflexiva) de diferentes países (Argentina, Brasil, Estados Unidos e Inglaterra). Esses terapeutas foram solicitados a descrever uma sessão ideal de psicoterapia de crianças classificando os itens do CPQ. Os modelos resultantes constituíram protótipos de cada uma das abordagens, e as análises estatísticas confirmaram a validade discriminante do instrumento.

A fidedignidade interavaliadores obtida com o CPQ vem sendo confirmada por vários estudos (Carvalho, Godinho, \& Ramires, 2016; Goodman \& Athey-Lloyd, 2011; Goodman et al., 2016; Ramires, Godinho, Carvalho, Gastaud, \& Goodman, 2017; Schneider, 2003; Schneider et al., 2010). Em todos eles, análises independentes de grupos de sessões de psicoterapia de crianças (variando de 20 a 53 sessões nos estudos) realizadas por avaliadores treinados têm atingido índices de concordância superiores a 0,70 (correlação intraclasse).

Goodman e Mavrides (2010) descreveram a análise de duas amostras de 33 vídeos de sessões realizadas por juízes independentes (correlação intraclasse $=0,71$, variando de 0,62 a 0,83). No estudo de Goodman e Athey-Lloyd (2011), 53 sessões filmadas foram analisadas (alpha de Cronbach $=0,77$, variando de 0,55 a 0,89 ). Semelhante resultado foi encontrado por Carvalho et al. (2016) e por Ramires et al. (2017), que analisaram 20 sessões das psicoterapias de duas crianças em idade escolar (alpha de Cronbach =0,75, variando de 0,70 a 0,81 em um caso; alpha de Cronbach = 0,74 , variando de 0,70 a 0,82 no outro caso). Além disso, o CPQ mostrou-se capaz de distinguir os tratamentos de dois diferentes pacientes com o mesmo terapeuta (Schneider et al., 2009) e dois tratamentos conduzidos por diferentes terapeutas 
com o mesmo paciente, sequencialmente (Goodman \& Athey-Lloyd, 2011).

\section{Procedimentos}

Inicialmente, a primeira autora deste artigo foi treinada pela autora do instrumento e por pesquisadores que vêm utilizando o CPQ em curso realizado no Anna Freud Centre, em Londres, durante um período de três dias. Uma segunda etapa do treinamento foi desenvolvida no Brasil, ministrada por um desses pesquisadores, durante uma semana. Obtida a certificação necessária, após a codificação de pelo menos dez sessões de psicoterapia com índices de concordância interavaliadores superiores a 0,70 , foi concedida a autorização para desenvolver e utilizar a versão em português do CPQ.

$\mathrm{O}$ projeto mais amplo no qual este estudo se insere foi analisado e aprovado pelo Comitê de Ética em Pesquisa da universidade da primeira autora. A tradução e adaptação do instrumento seguiram as diretrizes propostas por Borsa, Damásio e Bandeira (2012). Foram percorridas quatro etapas.

$\mathrm{Na}$ primeira etapa, foi realizada a tradução do CPQ do idioma de origem (inglês) para o idioma alvo (português), por duas tradutoras fluentes no idioma de origem e nativas no idioma alvo. As duas traduções foram realizadas de modo independente, sendo uma delas desenvolvida pela primeira autora deste artigo e a outra por psicóloga com experiência em tradução e sem conhecimento prévio do instrumento.

$\mathrm{Na}$ segunda etapa, foi realizada uma síntese das versões traduzidas. Cada item foi avaliado quanto à equivalência entre as versões traduzidas e o instrumento original. Essa avaliação foi pautada pelos parâmetros recomendados por Borsa et al. (2012), que sugerem avaliar a equivalência semântica, a equivalência idiomática, a equivalência experiencial e a equivalência conceitual. A versão sintetizada foi analisada e aprovada por um comitê de experts, formado por quatro psicólogos com experiência clínica que variava entre 2 e 32 anos (média $=21,5$ anos). Duas dessas psicólogas tinham treinamento e experiência prévia de utilização do PQS. Esta etapa incluiu a codificação de cinco sessões de psicoterapia com a versão sintetizada do CPQ. Borsa et al. (2012) propõem a realização, neste momento, de uma etapa para avaliação do instrumento pelo público-alvo. Como o CPQ é destinado a especialistas em psicoterapia, que atuam como juízes na codificação de sessões, essa etapa foi sobreposta à anterior, quando o comitê de experts analisou a versão traduzida e aplicou o instrumento em cinco sessões de psicoterapia.

Na terceira etapa, foi realizada a tradução reversa para o idioma de origem (back-translation). A versão sintetizada e revisada do $\mathrm{CPQ}$ foi retrotraduzida de maneira independente para o inglês por dois tradutores nativos no idioma alvo (português), fluentes e proficientes no idioma de origem do instrumento (inglês). A síntese das versões retrotraduzidas foi obtida por consenso entre esses dois tradutores e a versão final foi submetida à avaliação da autora do CPQ para aprovação final. O principal objetivo desta etapa foi analisar a equivalência conceitual dos itens que compõem o instrumento. Em uma quarta etapa, foi realizado um estudo piloto que envolveu a aplicação do CPQ a 20 sessões de duas psicoterapias de crianças. Os quatro clínicos que fizeram parte do comitê de experts participaram desse estudo piloto, que incluiu, ainda, uma etapa de treinamento para mais seis novos avaliadores. $\mathrm{O}$ treinamento foi coordenado pela primeira autora e envolveu a leitura e discussão do manual, a leitura conjunta dos itens do CPQ, a análise e discussão de cada um deles, a codificação conjunta de três sessões (ordenando os itens por consenso) e a codificação

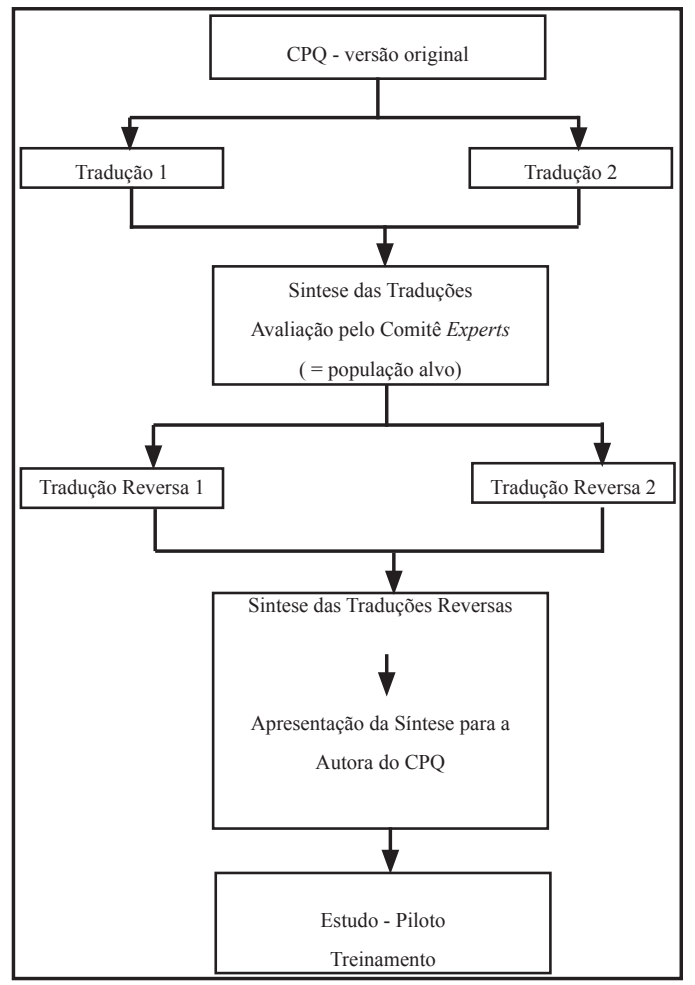

Figura 1. Etapas pecorridas para tradução e adaptação do CPQ

independente de sete sessões, sucedida pela discussão detalhada das categorias atribuídas para cada item. A Figura 1 sintetiza as etapas percorridas.

\section{Resultados}

A tabela 1 sintetiza os resultados das três primeiras etapas. A tabela mostra que houve uma boa equivalência na maioria dos itens do CPQ entre as versões dos dois tradutores na etapa de tradução do instrumento do idioma original para o idioma alvo (77\% dos itens). Em 15 casos, foi necessário realizar algum ajuste entre as traduções visando a uma formulação do item que assegurasse a sua equivalência semântica, isto é, a manutenção do significado do item ou o aprimoramento gramatical da tradução (Borsa et al., 2012). Em oito casos, foi necessário adaptar alguma expressão de difícil tradução - por exemplo, self-disclose, como mostra a tabela - por termos equivalentes na língua portuguesa, porém, sem que isso modificasse o significado do item.

$\mathrm{Na}$ tradução reversa para o idioma original do CPQ, aproximadamente $90 \%$ dos itens mantiveram-se inalterados, como se pode constatar na tabela 1. Pequenas alterações foram identificadas em dez itens, que não chegavam a 
Tabela 1. Equivalência dos Itens do CPQ na Tradução Inglês - Português e na Tradução Reversa

\begin{tabular}{|c|c|c|c|c|c|}
\hline \multicolumn{6}{|c|}{ Tradução Inglês - Português } \\
\hline \multirow{2}{*}{$\mathrm{E}$} & \multirow{2}{*}{$\mathrm{N}$} & \multicolumn{4}{|c|}{ Exemplos } \\
\hline & & Versão Original & Tradutor 1 & Tradutor 2 & Versão Sintetizada \\
\hline IE & 77 & Item 8: Child is curious. & A criança é curiosa. & A criança é curiosa. & A criança é curiosa. \\
\hline IES & 15 & $\begin{array}{l}\text { Item 9: Therapist is } \\
\text { nonresponsive. }\end{array}$ & $\begin{array}{l}\text { O terapeuta não é } \\
\text { responsivo. }\end{array}$ & O terapeuta é indiferente. & $\begin{array}{l}\text { O terapeuta não é } \\
\text { responsivo. }\end{array}$ \\
\hline IEI & 8 & $\begin{array}{l}\text { Item 21: Therapist self- } \\
\text { discloses. }\end{array}$ & O terapeuta faz revelações. & $\begin{array}{l}\text { O terapeuta se } \\
\text { autopromove. }\end{array}$ & $\begin{array}{l}\text { O terapeuta revela } \\
\text { informações sobre si. }\end{array}$ \\
\hline
\end{tabular}

\begin{tabular}{lllll}
\hline & & \multicolumn{3}{c}{ Tradução Reversa } \\
\hline
\end{tabular}

Nota: $\mathrm{E}$ = Resultados das Equivalências dos Itens; IE= Itens Equivalentes; IES = Itens ajustados por Equivalência Semântica; IEI = Itens ajustados por Equivalência Idiomática; IPA = Item Pouco Alterado; IMA = Item Muito Alterado

modificar o seu significado. Apenas em um caso (item 36), como mostra a tabela, a tradução reversa poderia dar margem a uma interpretação diferente do item original, o que demandou uma revisão dos tradutores e do comitê de experts.

A análise da autora do instrumento ratificou e aprovou a versão retrotraduzida. Alguns aspectos relativos à equivalência semântica e idiomática dos itens do instrumento foram discutidos. Por exemplo, na versão original do CPQ, em geral, não são utilizados os pronomes "a" ou "o" (Item 8 "Child is curious" ou Item 9 - "Therapist is nonresponsive"). Na versão final em português, optou-se por manter "A criança é curiosa" "O terapeuta não é responsivo" e assim por diante, respeitando as regras e estilo desta língua.

Com relação à equivalência conceitual, não foi identificado nenhum item cujo significado tivesse sido comprometido, tanto pelos profissionais que utilizaram o instrumento e atuaram como comitê de experts durante o processo ou na análise final da autora do CPQ. Quanto à equivalência experiencial (Borsa et al., 2012), constatou-se a aplicabilidade de todos os itens do CPQ no contexto brasileiro. Os itens desse instrumento são formulados de maneira clara, de fácil apreensão, podendo ser utilizados por pesquisadores e clínicos cujo trabalho é alicerçado em distintas abordagens psicoterápicas, uma vez que não são saturados teoricamente. Os itens do CPQ descrevem comportamentos e atitudes passíveis de serem observados em sessões de psicoterapia de crianças em geral, independentemente do contexto social e cultural. Sua presença ou ausência, em maior ou menor intensidade, é justamente o que interessa para a análise do processo com aquela criança em particular e aquela díade, de acordo com a idade, o quadro clínico da criança e a etapa da psicoterapia.

Durante o treinamento e o estudo piloto, algumas observações foram feitas com relação ao manejo inicial do instrumento. Nas primeiras sessões avaliadas, houve uma tendência dos juízes de interpretar o material da sessão e de avaliar ou criticar as intervenções do terapeuta. Constatou-se que a vivacidade da filmagem de uma sessão terapêutica impacta de modo diferente e mais intenso o observador externo do que o relato de uma sessão sintetizada ou dialogada por escrito, procedimento mais comumente utilizado no nosso meio tanto para fins de supervisão clínica como de pesquisa.

Contudo, o propósito do CPQ, que possibilita a investigação naturalística da psicoterapia com crianças, é descrever e analisar seu processo tal como ocorre em cada sessão. O manual orienta o avaliador no sentido de uma observação neutra do vídeo, identificando o que foi mais saliente e característico e o que não foi, pontuando os itens de acordo com essa observação.

Outro aspecto discutido no estudo piloto e no treinamento foi a diferença, inicialmente existente, na forma de avaliar dos juízes com menos experiência clínica e daqueles com mais tempo de trabalho como psicoterapeutas. Contrariamente ao que se esperava, os terapeutas menos experientes atingiram níveis bastante satisfatórios de concordância interavaliadores desde as primeiras sessões analisadas, diferentemente dos juízes mais experientes. No primeiro caso, observou-se que os terapeutas estavam menos impregnados teoricamente, mais livres de pré-concepções, assimilando mais rapidamente o procedimento proposto pelo instrumento.

As duas psicoterapeutas que já tinham experiência com o PQS (a forma adulta do instrumento) e que integraram o comitê de experts consideraram o CPQ mais complexo e difícil de utilizar. Possivelmente, isso se deve mais às características da psicoterapia de crianças, comparada à do adulto, do que ao instrumento propriamente dito. A sessão terapêutica do adulto centra-se majoritariamente na comunicação verbal. Em contrapartida, a sessão terapêutica das crianças envolve, além da comunicação verbal, diversas 
outras possibilidades que incluem significativamente o comportamento não verbal, o brincar, o não brincar, os jogos, etc.

O treinamento, e as discussões realizadas durante o seu curso, contribuiu para a atenuação dos efeitos descritos acima e para sua superação. Ocupando aproximadamente 40 horas e um total de 6 semanas, a diferença nos resultados obtidos entre a primeira e a décima sessão codificada testemunha essa mudança. Deve-se salientar que as sessões avaliadas foram selecionadas aleatoriamente e que os juízes estavam cegos para quaisquer informações sobre as crianças e seu quadro clínico. O método de correlações intraclasses indicou uma média na concordância interavaliadores de 0,579 na primeira sessão codificada (alpha de Cronbach), variando de 0,220 a 0,720 , e uma média de 0,802 na décima sessão codificada durante o treinamento, variando de 0,741 a 0,835. A versão em português dos 100 itens do CPQ é apresentada no Apêndice.

\section{Discussão}

A maioria dos ajustes necessários na etapa de tradução do CPQ para o idioma alvo foi direcionada para a equivalência semântica, visando garantir o uso de palavras com o mesmo significado no idioma original e a correção de erros gramaticais da tradução. Apenas em oito casos foi necessário realizar ajustes no sentido de assegurar a equivalência idiomática dos itens. No seu conjunto, a versão em português do instrumento apresentou boa equivalência experiencial e conceitual.

As características das sessões de psicoterapia de crianças, os fenômenos presentes e os conceitos que alicerçam essa prática clínica foram representados de modo abrangente e suficiente na versão original do CPQ. Borsa et al. (2012) alertam para a importância de que tais conceitos e domínios sejam pertinentes e apreendidos na nova cultura da população alvo. O processo de tradução e adaptação do CPQ para o português permitiu constatar sua adequação e pertinência para o estudo do processo psicoterapêutico de crianças em nosso contexto.

Diferentemente do estudo de Serralta et al. (2007) com o PQS, no qual não foi constatada nenhuma tendência nas avaliações relacionada à experiência clínica dos avaliadores, no presente estudo, houve uma dificuldade maior dos juízes mais experientes, na avaliação das primeiras sessões, de manter um distanciamento ótimo do material analisado e de descrever a sessão ao invés de interpretá-la. Uma possível explicação para esse fato é que vários desses juízes eram clínicos, mais do que pesquisadores, o que pode ter impactado, em alguma medida, em uma etapa em que ainda não estavam familiarizados com os procedimentos propostos pelo CPQ, sua prontidão para apenas codificar os itens de acordo com o que observaram nos vídeos, seguindo rigorosamente as orientações do manual, ao invés de considerar como teriam agido ou como entendiam que o terapeuta deveria fazê-lo.

A questão da filmagem das sessões também deve ser discutida. Trata-se de um procedimento controverso, especialmente nas abordagens de tradição psicanalítica, por constituir-se como um dispositivo importante no setting clínico e potencialmente impactante para a relação terapeutapaciente e para cada um deles em particular. Todavia, esse procedimento vem sendo largamente utilizado em estudos desenvolvidos nos Estados Unidos, Canadá e em diversos países da Europa.

Estudos de processo, com a abrangência e profundidade necessárias, baseados em relatos escritos da sessão, tornamse significativamente limitados e enviesados. Nas narrativas baseadas na memória do terapeuta, o relato sofre a ação do processo secundário e constitui uma versão revisada e censurada dos eventos da sessão (Serralta et al., 2007). Além disso, em geral, os relatos de sessões e seu uso em supervisões e seminários clínicos não costumam ser comunicados ao paciente (contrariando o contrato de sigilo), em oposição à filmagem, cujo uso é condicionado aos esclarecimentos sobre sua finalidade e ao consentimento da criança e de seus pais. Esse uso torna-se plenamente assimilado e incorporado no setting terapêutico, como se constatou neste estudo.

A familiaridade crescente dos juízes com os itens do CPQ conduziu a uma agilidade cada vez maior na codificação dos itens do instrumento e a uma habilidade maior no julgamento da pontuação que deveria ser atribuída. Por exemplo, em relação ao item 46 - "O terapeuta interpreta o significado do jogo da criança", se durante a sessão o terapeuta realizar uma única interpretação e deixar de fazê-lo em vários outros momentos em que o jogo é claramente vinculado a eventos, situações ou sentimentos significativos e explícitos (como descreve o manual), esse item receberá uma pontuação baixa (categoria 1, 2 ou 3) ou neutra (categoria 4, 5 ou 6), ao invés de uma pontuação alta (categoria 7, 8 ou 9), de acordo com a distribuição dos outros itens e com o quanto cada um deles houver sido mais ou menos saliente e mais ou menos significativo.

Como descrito acima, os itens do CPQ foram formulados com base em extensa revisão de literatura sobre psicoterapia de crianças, instrumentos sobre processo existentes e adaptações da forma adulta PQS (Schneider, 2003; Schneider \& Jones, 2006; Schneider et al., 2009). Isso faz com que, no seu conjunto, eles possam ser representativos de diferentes abordagens psicoterápicas. Estudo recente realizado com especialistas em diferentes modalidades de tratamento com crianças resultou na identificação de protótipos de psicoterapia psicodinâmica, terapia cognitivocomportamental e abordagem baseada na função reflexiva (Goodman et al., 2016). Nesses modelos, os itens considerados mais característicos de uma sessão ideal com crianças foram identificados, permitindo uma análise da adequação entre a psicoterapia proposta e aquela que efetivamente acontece, a detecção do uso de técnicas mistas, a detecção dos fatores comuns às diferentes abordagens e o reconhecimento daquilo que costuma ser mais característico em diferentes quadros clínicos e com crianças de diferentes idades.

Todas essas alternativas abrem um leque interessante de possibilidades para a compreensão do processo da psicoterapia e suas variações no interior do caso individual e entre diferentes casos clínicos. O CPQ pode ser utilizado em diversos delineamentos de pesquisa, tanto em estudos de caso único como em estudos de casos múltiplos.

A necessidade de um treinamento sistemático e o considerável tempo que sua utilização requer podem ser 
aspectos vistos como objeções à sua utilização. De fato, tais demandas precisam ser consideradas ao optar-se pelo uso do instrumento. Por outro lado, os estudos de processo terapêutico dificilmente escapam da complexidade dos procedimentos para sua realização, dada sua natureza.

As etapas percorridas para a adaptação desse instrumento para nosso contexto, incluindo o estudo piloto, permitiram confirmar suas vantagens, anteriormente destacadas por Schneider (2003):

1. O grande número de itens do CPQ permite descrições de uma larga extensão de fenômenos, capturando eventos salientes do processo terapêutico;

2. As características do instrumento e sua forma de categorização permitem uma descrição do processo ao longo do tempo e não apenas de um segmento da sessão ou do tratamento;

3. O CPQ é compatível com diferentes pontos de vista teóricos, dada a formulação panteórica dos Q-itens;

4. As regras explícitas do Manual oferecem diretrizes que previnem o uso de inferências. A codificação baseia-se nas pistas e sinais comportamentais e linguísticos, favorecendo um julgamento mais objetivo;

5. O fato de os itens serem codificados em um continuum e não em uma categorização sim ou não ou do tipo ou ou previne alguns vieses.

OCPQ permite o desenvolvimento de estudos combinados de processo e resultados. A codificação de todas as sessões de um tratamento, ou de um grande e representativo número delas, e a aplicação de medidas repetidas de resultado permite análises que possam estabelecer conexões entre o processo e as mudanças obtidas durante o tratamento. Isso é importante porque adiciona elementos para que se compreenda o que funciona e o que não funciona em uma psicoterapia de crianças, bem como sobre quais são os seus mecanismos de mudança (Kazdin, 2009).

Estudos futuros podem contribuir para elucidar as evidências de validade do instrumento, por exemplo, sua validade de conteúdo, validade discriminante e estabilidade temporal (Borsa et al., 2012). O uso mais abrangente do CPQ em nossa cultura poderia contribuir, também, para uma revisão do manual e das vinhetas ilustrativas, minimizando eventuais dificuldades encontradas na codificação de alguns itens.

O uso do CPQ em novas investigações poderá possibilitar a identificação das estruturas de interação terapeutapaciente ao longo do tratamento (Jones, 2000) e a relação de tais estruturas com os resultados (Ablon \& Jones, 2005; Goodman, Edwards, \& Chung, 2014; Jones, 2000). Explorar diferenças no processo terapêutico entre pacientes, terapeutas, mudanças nos itens ao longo do tempo de um tratamento, mudanças nos itens como função do resultado, o grau de adesão aos protótipos ou modelos teóricos, as mudanças nessa adesão ao longo de uma psicoterapia são possibilidades oferecidas pelo instrumento com potencial de contribuir para a ampliação do conhecimento acerca dos processos envolvidos na psicoterapia de crianças.

\section{Referências}

Ablon, S. J., \& Jones, E. (2005). On analytic process. Journal of American Psychoanalytic Association, 53(2), 541-568. doi: 10.1177/00030651050530020101

Borsa, J. C., Damásio, B. F., \& Bandeira, D. R. (2012). Adaptação e validação de instrumentos psicológicos entre culturas: Algumas considerações. Paidéia, 22(53), 423-432. doi: http://dx.doi. org/10.1590/S0103-863X2012000300014

Carvalho, C., Godinho, L. R., \& Ramires, V. R R. (2016). O processo psicoterapêutico de uma criança: análise baseada no Child Psychotherapy Q-Set. Temas em Psicologia, v. 24, p. 1153-1167.

Fonagy, P. (2003). The research agenda: The vital need for empirical research in child psychotherapy. Journal of Child Psychotherapy, 29(2), 129-136. doi: 10.1080/0075417031000138406

Fonagy, P. (2009). Research in child psychotherapy: Progress, problems and possibilities?. In N. Midgley, J. Anderson, E. Grainger, T. Vuckovic-Nesic, \& C. Urwin (Eds.), Child psychotherapy and research: New approaches, emerging findings. (pp. 19-34). Nova York, NY: Routledge.

Goodman, G., \& Athey-Lloyd, L. (2011). Interaction structures between a child and two therapists in the psychodynamic treatment of a child with Asperger's disorder. Journal of Child Psychotherapy, 37(3), 311-326.doi:10.1080/007541 7X.2011.614749

Goodman, G., Edwards, K., \& Chung, H. (2014). Interaction structures formed in the psychodynamic therapy of five patients with borderline personality disorder in crisis. Psychology and Psychotherapy: Theory, Research and Practice, 87, 15-31. doi:10.1111/papt.12001

Goodman, G., \& Mavrides, L. (2010). Countertransference, process, and outcome in psychodynamic child psychotherapy. Paper presented at the meeting of the Society for Psychotherapy Research, Asilomar, Califórnia.

Goodman, G., Midgley, N., \& Schneider, C. (2016). Expert clinicians' prototypes of an ideal child treatment in psychodynamic and cognitive-behavioral therapy: Is mentalization seen as a common process factor? Psychotherapy Research, 26, 590-601.

Jones, E. (2000). Therapeutic action. A guide to psychoanalytic therapy. London: Jason Aronson.

Jones, E., Cumming, J. D., \& Horowitz, M. J. (1988). Another look at the nonspecific hypothesis of therapeutic effectiveness. Journal of Consulting Clinical Psychology, 56(1), 48-55.

Jones, E., \& Pulos, S. M. (1993). Comparing process in psychodynamic and cognitive-behavioral therapies. Journal of Consulting Clinical Psychology, 61(2), 306-16.

Kazdin, A. E. (2000). Psychotherapy for children and adolescents: Directions for research and practice. New York: Oxford University Press.

Kazdin, A. E. (2008). Evidence-based treatment and practice. New opportunities to bridge clinical research and practice, enhance the knowledge base, and improve patient care. American Psychologist, 63(3), 146-159.

Kazdin, A. E. (2009). Understanding how and why psychotherapy leads to change. Psychotherapy Research, 19(4-5), 418-428.

Midgley, N. (2007). Researching the process of psychoanalytic child psychotherapy. In E. Kennedy \& N. Midgley (Eds), Process and outcome research in child, adolescent and parent-infant psychotherapy: A thematic review (pp. 8-53). London: North Central London Strategic Health Authority. 
Ramires, V. R. R., Godinho, L. R., Carvalho, C., Gastaud, M., \& Goodman, G. (2017). Child psychoanalytic psychotherapy: a single case study. Psychoanalytic Psychotherapy, v. 31, p. 1-19..

Schneider, C. (2003). The development of the Child Psychotherapy $Q$-set (Doctoral Dissertation). University of California, Berkeley.

Schneider, C., \& Jones, E. E. (2006). Child Psychotherapy Q-Set. Coding manual (Unpublished Manual). Berkeley: University of California.

Schneider, C., \& Jones, E. E. (2012). Appendix IB. Child Psychotherapy Q-Set. Coding manual. In R. A. Levy, J. S. Ablon \& H. Kächele (Eds), Psychodynamic psychotherapy research: Evidence-based practice and practice-based evidence (pp 611-626). New York: Humana Press.
Schneider, C., Midgley, N., \& Duncan, A. (2010). A “motion portrait" of a psychodynamic treatment of an 11-year-old girl: Exploring interrelations of psychotherapy process and outcome using the Child Psychotherapy Q-Set. Journal of Infant, Child and Adolescent Psychotherapy, 9, 94-107. doi: 10.1080/15289168.2010.510979.

Schneider, C., Pruetzel-Thoma, A., \& Midgley, N. (2009). Discovering new ways of seeing and speaking about psychotherapy process: The Child Psychotherapy Q-Set. In N. Midgley, J. Anderson, E. Grainger, T. Vuckovic-Nesic, \& C. Urwin (Eds.), Child psychotherapy and research: New approaches, emerging findings (pp. 72-84). Nova York, NY: Routledge.

Serralta, F., Nunes, M. L. T., \& Eizirik, C. (2007). Elaboração da versão em português do Psychotherapy Process Q-set. Revista de Psiquiatria do Rio Grande do Sul, 29(1), 44-55. Retrieved from http://www.scielo.br/pdf/rprs/v29n1/v29n1a11.pdf

Stephenson, W. (1982). Q-Methodology, interbehavioral psychology, and quantum theory. The Psychological Record, $32,235-248$. 


\section{Apêndice \\ Child Psychotherapy Q-Set - Versão em Português}

Item 1: A criança expressa sentimentos negativos (ex. crítica, hostilidade) em direção ao terapeuta [vs. expressa aprovação ou admiração].

Item 2: O terapeuta comenta sobre o comportamento não verbal da criança (ex. postura corporal, gestos).

Item 3: Os comentários do terapeuta visam a encorajar a fala da criança.

Item 4: Há discussão sobre por que a criança está em terapia.

Item 5: A criança tem dificuldade para compreender os comentários do terapeuta.

Item 6: O terapeuta é sensível aos sentimentos da criança.

Item 7: A criança está ansiosa e tensa [vs. calma e descontraída].

Item 8: A criança é curiosa.

Item 9: O terapeuta não é responsivo [vs. afetivamente engajado].

Item 10: A criança procura maior intimidade com o terapeuta.

Item 11: Sentimentos ou pensamentos sexuais emergem.

Item 12: O terapeuta modela emoções não ditas ou não elaboradas.

Item 13: A criança está animada ou excitada.

Item 14: Sintomas físicos ou a saúde são discutidos.

Item 15: A criança faz contato físico com o terapeuta.

Item 16: Há discussão ou evidência de funções corporais (ex. movimentos intestinais).

Item 17: O terapeuta ativamente exerce controle sobre a interação (ex. estruturando, introduzindo novos tópicos).

Item 18: O terapeuta é crítico e transmite falta de aceitação.

Item 19: A criança pede conselhos ou informações.

Item 20: A criança é provocadora; desafia o terapeuta ou as regras e limites da sessão.

Item 21: O terapeuta revela informações sobre si.

Item 22: A criança expressa medos de ser punida ou ameaçada.

Item 23: A sessão terapêutica tem um tema ou foco específico.

Item 24: Conflitos emocionais do terapeuta invadem o relacionamento.

Item 25: A criança tem dificuldade de encerrar a sessão.

Item 26: A criança é socialmente não sintonizada ou inapropriada.

Item 27: Há um foco em ajudar a criança a planejar seu comportamento fora da sessão.

Item 28: O terapeuta percebe acuradamente o processo terapêutico.

Item 29: A qualidade do brincar da criança é fluido, absorto [vs. fragmentado, esporádico].

Item 30: As aspirações ou ambições da criança são temas.

Item 31: O terapeuta solicita mais informação ou elaboração.

Item 32: A criança alcança uma nova compreensão ou insight.

Item 33: A criança expressa sentimentos sobre a necessidade de alguém, ou estar próxima de alguém.

Item 34: A criança culpa os outros, ou forças externas, pelas dificuldades.

Item 35: A autoimagem da criança é um tema.

Item 36: O terapeuta assinala o uso de defesas por parte da criança.

Item 37: O terapeuta se comporta de uma maneira didática.

Item 38: O terapeuta e a criança demontram um vocabulário ou compreensão compartilhada quando se referem a eventos ou sentimentos.

Item 39: A criança é competitiva, rivaliza com o terapeuta.

Item 40: A criança se comunica sem afeto.

Item 41: A criança não se sente compreendida pelo terapeuta.

Item 42: A criança ignora ou rejeita os comentários e observações do terapeuta.

Item 43: O terapeuta sugere o significado do comportamento dos outros.

Item 44: A criança se sente cautelosa ou desconfiada (vs. confiante e segura).

Item 45: O terapeuta tolera os fortes afetos ou impulsos da criança.

Item 46: O terapeuta interpreta o significado do jogo da criança.

Item 47: Quando a interação com a criança é difícil, o terapeuta tenta se ajustar à criança.

Item 48: O terapeuta coloca limites.

Item 49: A criança transmite ou expressa sentimentos confusos ou conflituosos sobre o terapeuta.

Item 50: O terapeuta chama a atenção para os sentimentos considerados inaceitáveis pela criança (ex. raiva, inveja, ou excitação).

Item 51: A criança atribui as próprias características ou sentimentos ao terapeuta.

Item 52: O terapeuta faz afirmações explícitas sobre o final da sessão, o próximo final de semana ou as férias.

Item 53: A criança expressa consciência das próprias dificuldades internas.

Item 54: A criança é clara e organizada na sua expressão verbal.

Item 55: O terapeuta recompensa diretamente comportamentos desejáveis.

Item 56: A criança está distante dos seus sentimentos.

Item 57: O terapeuta tenta modificar distorções nas crenças da criança.

Item 58: A criança parece relutante em examinar pensamentos, reações ou motivações relacionadas aos problemas.

Item 59: A criança se sente inadequada e inferior [vs. eficaz e superior]. 
Item 60: $\mathrm{O}$ terapeuta é protetor em relação à criança.

Item 61: A criança se sente tímida e envergonhada [vs. desinibida e segura].

Item 62: O terapeuta assinala um tema recorrente na experiência ou conduta da criança.

Item 63: A criança explora relacionamentos com outros significativos.

Item 64: A criança inclui o terapeuta no jogo.

Item 65: O terapeuta clarifica, reafirma, ou reformula comunicações da criança.

Item 66: O terapeuta é diretamente tranquilizador.

Item 67: O terapeuta interpreta os desejos, sentimentos ou ideias rejeitados ou inconscientes.

Item 68: Os significados reais da experiência são ativamente diferenciados dos fantasiados.

Item 69: A situação de vida atual ou recente da criança é enfatizada.

Item 70: A criança se esforça para controlar sentimentos ou impulsos.

Item 71: A criança se engaja no jogo de faz-de-conta.

Item 72: A criança é ativa.

Item 73: A criança expressa medos ou exibe um comportamento fóbico.

Item 74: O humor é utilizado.

Item 75: Interrupções, pausas no tratamento ou o término da terapia são discutidos.

Item 76: $\mathrm{O}$ terapeuta faz ligações entre sentimentos e experiências da criança.

Item 77: A interação do terapeuta com a criança é sensível ao seu nível de desenvolvimento.

Item 78: A criança é complacente.

Item 79: O terapeuta comenta as mudanças no humor ou nas emoções da criança.

Item 80: A criança se comporta de uma maneira dependente [vs. insiste na independência].

Item 81: O terapeuta enfatiza sentimentos para ajudar a criança a experimentá-los mais profundamente.

Item 82: O terapeuta ajuda a criança a manejar os sentimentos.

Item 83: A criança é exigente.

Item 84: A criança expressa raiva ou sentimentos agressivos.

Item 85: A agressividade da criança é direcionada para ela mesma.

Item 86: O terapeuta é confiante, seguro de si [vs. incerto ou inseguro].

Item 87: O terapeuta informa a criança do impacto potencial do seu comportamento sobre os outros (não incluindo o terapeuta).

Item 88: O material da sessão é significativo e relevante em relação aos conflitos da criança.

Item 89: $\mathrm{O}$ terapeuta age para fortalecer as defesas existentes.

Item 90: Os sonhos ou fantasias da criança são discutidos.

Item 91: Uma fase do desenvolvimento anterior é um tópico.

Item 92: Os sentimentos ou percepções da criança são relacionados a situações ou comportamentos do passado.

Item 93: O terapeuta é neutro.

Item 94: A criança se sente triste ou deprimida [vs. animada e alegre].

Item 95: O brincar da criança carece de espontaneidade.

Item 96: Os pais da criança são um tópico de discussão.

Item 97: O terapeuta enfatiza a verbalização dos estados internos e afetos.

Item 98: A relação terapêutica é discutida.

Item 99: O terapeuta oferece ajuda ou orientação.

Item 100: O terapeuta faz conexões entre a relação terapêutica e outros relacionamentos. 